\title{
HISTOCYTOPATHOLOGICAL DIAGNOSIS OF ROSAI-DORFMAN DISEASE: CASE REPORT AND LITERATURE REVIEW
}

Zephania Abraham ${ }^{1}$, Zerd Francis ${ }^{2}$, Advera Ngaiza $^{3}$, Aveline Kahinga ${ }^{4}$, and Faustine Bukanu $^{2}$

${ }^{1}$ University of Dodoma College of Health and Allied Sciences

${ }^{2}$ Benjamin Mkapa Hospital

${ }^{3}$ Muhimbili National Hospital

${ }^{4}$ Muhimbili University of Health and Allied Sciences School of Medicine

July 29, 2021

\author{
Abstract \\ Sinus histiocytosis with massive lymphadenopathy (Rosai-Dorfman disease) being a rare benign proliferative self-limiting disease \\ of the cells of macrophage-histiocyte family is of unknown etiology and presents with massive lymphadenopathy. We are \\ hereby reporting a case of RDD presenting with massive bilateral cervical and submandibular lymphadenopathy, diagnosed by \\ histocytopathology. \\ HISTOCYTOPATHOLOGICAL DIAGNOSIS OF ROSAI-DORFMAN DISEASE: CASE RE- \\ PORT AND LITERATURE REVIEW \\ Zephania Saitabau Abraham ${ }^{1}$ Zerd Francis ${ }^{2}$ Advera Isaac Ngaiza ${ }^{3}$ Aveline Aloyce Kahinga \\ ${ }^{4}$ Faustine Bukanu ${ }^{5}$ \\ ${ }^{1}$ Department of Surgery-University of Dodoma, School of Medicine and Dentistry, Dodoma-Tanzania \\ 2 Department of Pathology-The Benjamin Mkapa Hospital-Dodoma, Tanzania \\ ${ }^{3}$ Department of Anatomical Pathology-Muhimbili National Hospital, Dar es Salaam, Tanzania \\ ${ }^{4}$ Department of Otorhinolaryngology-Muhimbili University of Health and Allied Sciences, Dar es Salaam- \\ Tanzania \\ ${ }^{5}$ Department of Otorhinolaryngology-The Benjamin Mkapa Hospital, Dodoma-Tanzania \\ Correspondence \\ Dr. Zephania Saitabau Abraham \\ Department of Surgery- University of Dodoma, \\ School of Medicine and Dentistry \\ Email: zsaitabau@yahoo.com
}

\section{ABSTRACT}


Sinus histiocytosis with massive lymphadenopathy (Rosai-Dorfman disease) being a rare benign proliferative self-limiting disease of the cells of macrophage-histiocyte family is of unknown etiology and presents with massive lymphadenopathy. We are hereby reporting a case of RDD presenting with massive bilateral cervical and submandibular lymphadenopathy, diagnosed by histocytopathology.

KEYWORDS: Histocytopathology, Rosai-Dorfman, Tanzania.

\section{KEY CLINICAL MESSAGE}

Being a rare disease, establishing the diagnosis of Rosai-Dorfman Disease requires a high index of suspicion by pathologist so as to differentiate from other possible causes of massive cervical and submandibular lymphadenopathy

\section{Introduction}

Sinus histiocytosis with massive lymphadenopathy (SHML) also known as Rosai-Dorfman disease is a benign, self-limiting disease that commonly involve lymph nodes. ${ }^{1-6}$ The disease was first described by Rosai and Dorfman in $1969^{7,8}$ and since then, there has been some many more cases reported from the available literatures.

In terms of age and sex predilection, RDD commonly affect children and young adult in their second decades life and shows slight male preponderance. ${ }^{9-12}$

RDD may show an extra nodal disease pattern (23\%) particularly by having predilection to the head and neck region ( $75 \%$ of cases $)^{4,13,14}$ and involvement of [?] 1 extra nodal site has been identified in $43 \%$ of cases. ${ }^{13}$ The tendency of RDD to have simultaneous involvement of nodal and extranodal sites remains to be rare and its diagnosis may be challenging. ${ }^{12}$

SHML being a newly recognized and distinct pseudolymphomatous benign entity has distinctive microscopic features though its histocytomorphology has been documented in relatively few cases. ${ }^{15}$

SHML has several clinical features that include massive painless, bilateral, cervical lymphadenopathy which may be accompanied by leukocytosis, fever, elevated erythrocyte sedimentation rate (ESR), and hypergammaglobulinemia. ${ }^{1,4-6,8,10,13,16}$

In extra nodal cases though rare, SHML shows a distinct predilection to the head and neck region ${ }^{4,14}$ and involved extra nodal sites include the eyes and ocular adnexa, head and neck, upper respiratory tract, skin, subcutaneous tissue, bone, skeletal muscle, central nervous system, gastrointestinal tract, salivary glands, genitourinary tract, thyroid, breast, liver, kidney, heart, uterine cervix. ${ }^{1,4,5,14}$ Misdiagnosis sometimes may be encountered in patients with massive lymphadenopathy since they may be labeled to be having lymphomas or other neoplasms depending on the affected anatomical sites such as involvement of the nose and paranasal sinuses by sinus histiocytosis. ${ }^{10,12,17}$

To date, the implicated etiological agent for RDD remains to be unknown though there are several postulated theories to explain its aetiopathogenesis. An autoimmune phenomenon (in about 10-12\% of patients) has been reported to be one of the aetiology. ${ }^{13}$ Some infectious agents have also been postulated in causation such as Polyomavirus, Epstein-Barr virus, Parvovirus B19, Herpes virus type 6 and 8. ${ }^{10,13}$ Similarly, some relationship with microorganisms such as Klebsiella, Brucella and Cytomegalovirus has also been postulated in its causation ${ }^{10,12,13}$ though any attempt to isolate such organisms consistently failed. ${ }^{13}$

Other postulated mechanisms in pathogenesis of RDD include immune dysfunction or an aberrant exaggerated immune response to an infectious agent or an antigen that can cause proliferation of histiocytes and similarly, stimulation of monocytes/macrophage via macrophage colony-stimulating factor has also been postulated in causation ${ }^{8,12,13}$ thus suggesting immune system dysregulation in patients with RDD.

We report such a case of Rosai-Dorfman Disease diagnosed by FNAC and excisional biopsy with emphasis on clue to histocytopathological interpretation. 


\section{Case presentation}

We are presenting a 58-year-old Tanzanian female patient who presented at our outpatient otorhinolaryngology clinic at Benjamin Mkapa Hospital with a 6-months history of low-grade fever and bilateral cervical and submandibular lymph nodes.

On physical examination, the patient was found to have bilateral cervical and submandibular lymphadenopathy and occasional low-grade fevers. Local examination revealed multiple nodes with the largest one measuring $5 \times 5 \mathrm{~cm}$, ovoid, mobile in all directions and non-tender. Laboratory results showed hemoglobin $11 \mathrm{~g} / \mathrm{dl}$ and elevated ESR (30mm/hour) and a diagnosis of probable lymphoma was made. FNAC was ordered that revealed a smear (hematoxylin and eosin stain used) showing histiocytes with emperipolesis, scattered lymphoglandular bodies and also atypical histiocytes with dense inflammatory infiltrates were noted suggestive of RDD. (Figures 1, 2 and 3)

Figure 1 : Cytology smear showing histiocytes with emperipolesis, lymphocytes and scattered lymphoglandular bodies.

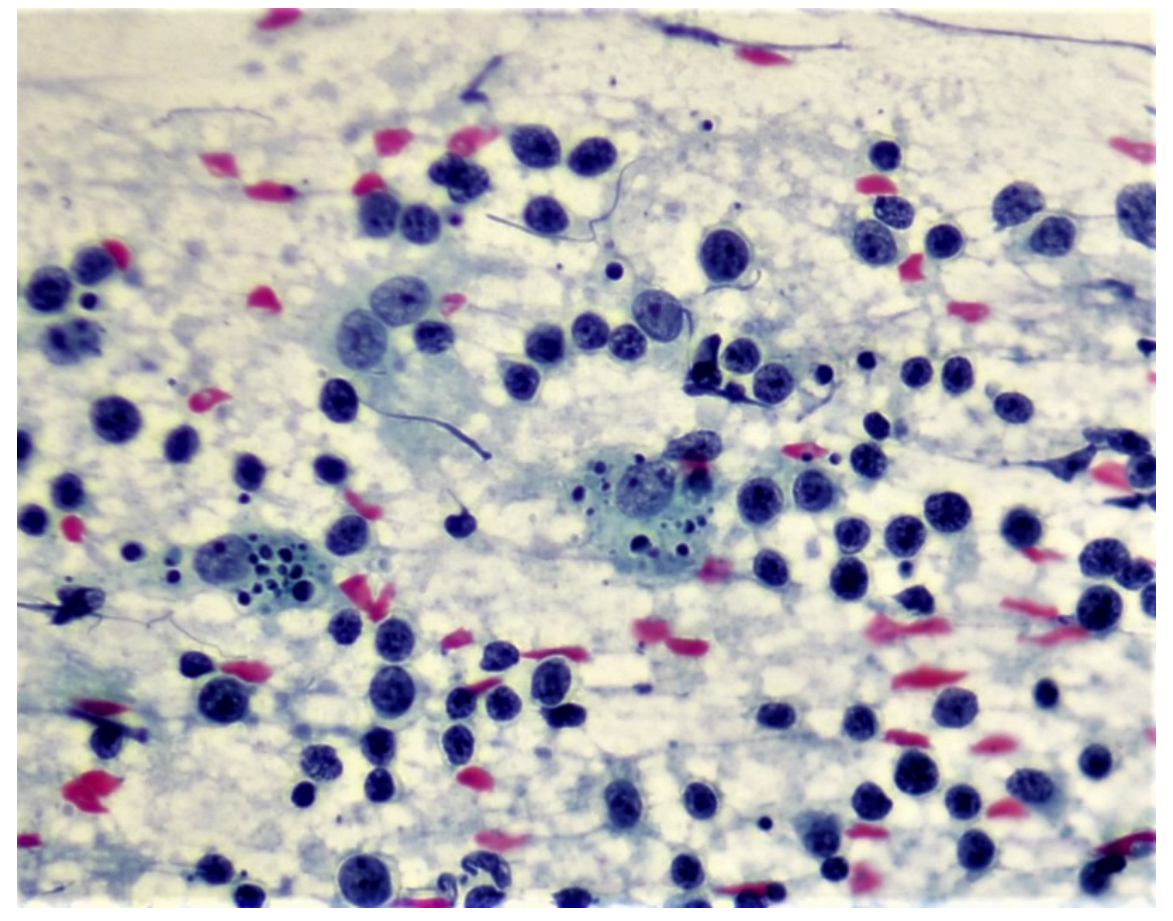

Figure 2: Cytology smear showing histiocytes with emperipolesis, some histiocytes showed multinucleation. 


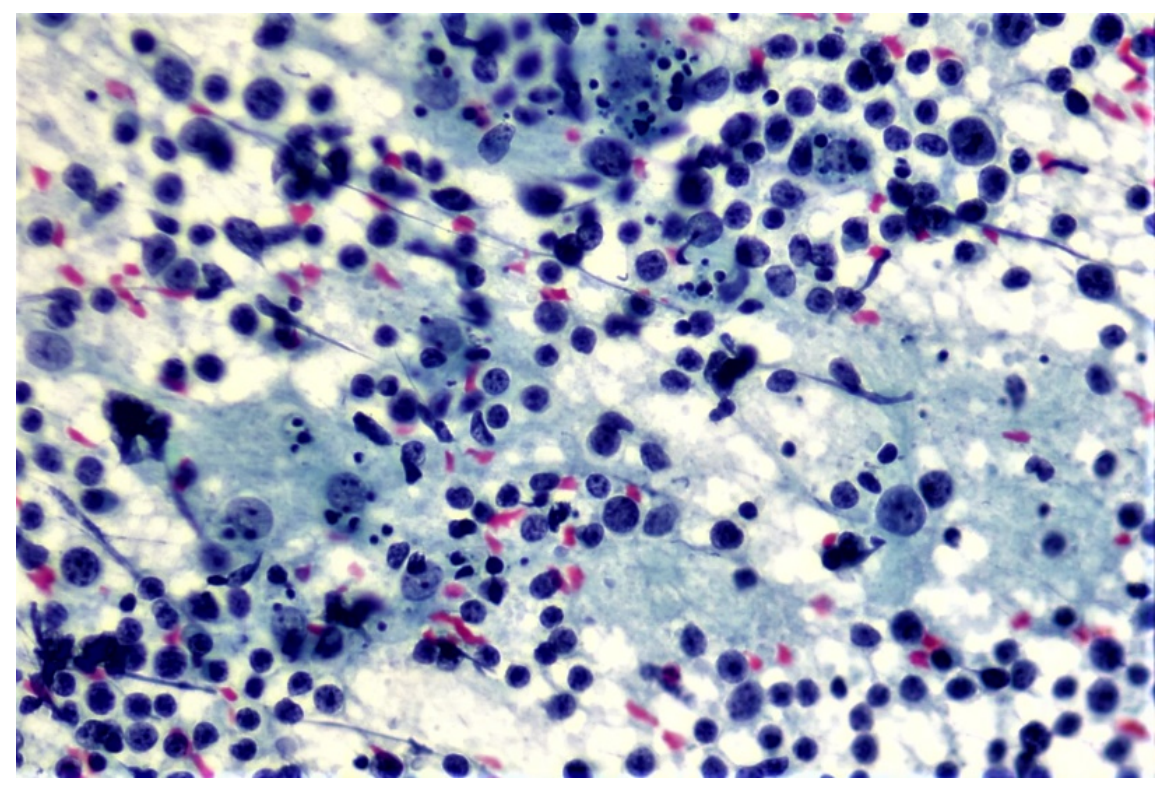

Figure 3: A cytologic smear showing histiocytes with dense inflammatory infiltrates in a cytological smear

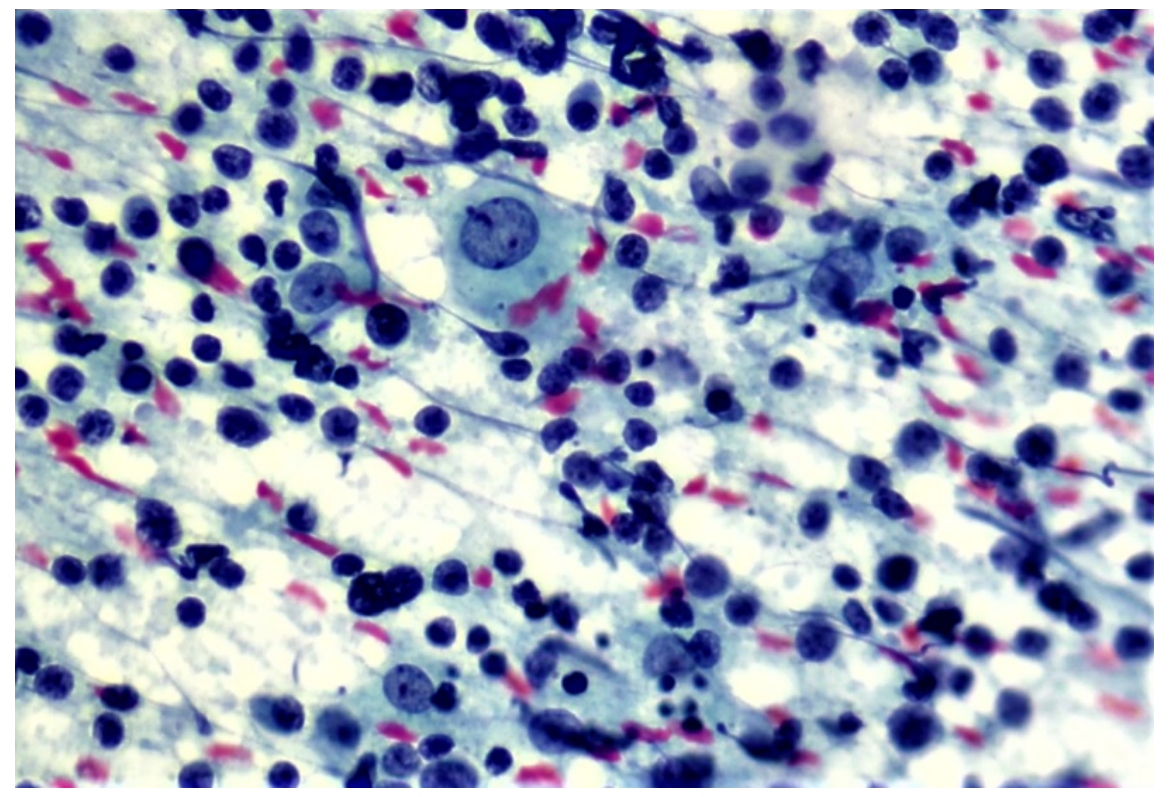

Open lymph node biopsy was then done and the smear revealed a fragment composed of dilated sinuses filled with large histiocytes with intact lymphocytes and plasma cells (emperipolesis, lymphophagocytosis) with large round, vesicular nuclei with delicated nuclear membrane and prominent nucleoli, some histiocytes showed multinucleation. Elsewhere, mixed inflammatory cells noted. Confirmatory diagnosis of RDD was made.

(Figure 4 and 5)

Figure 4: Atypical histiocytes with inflammatory infiltrates in histological section 


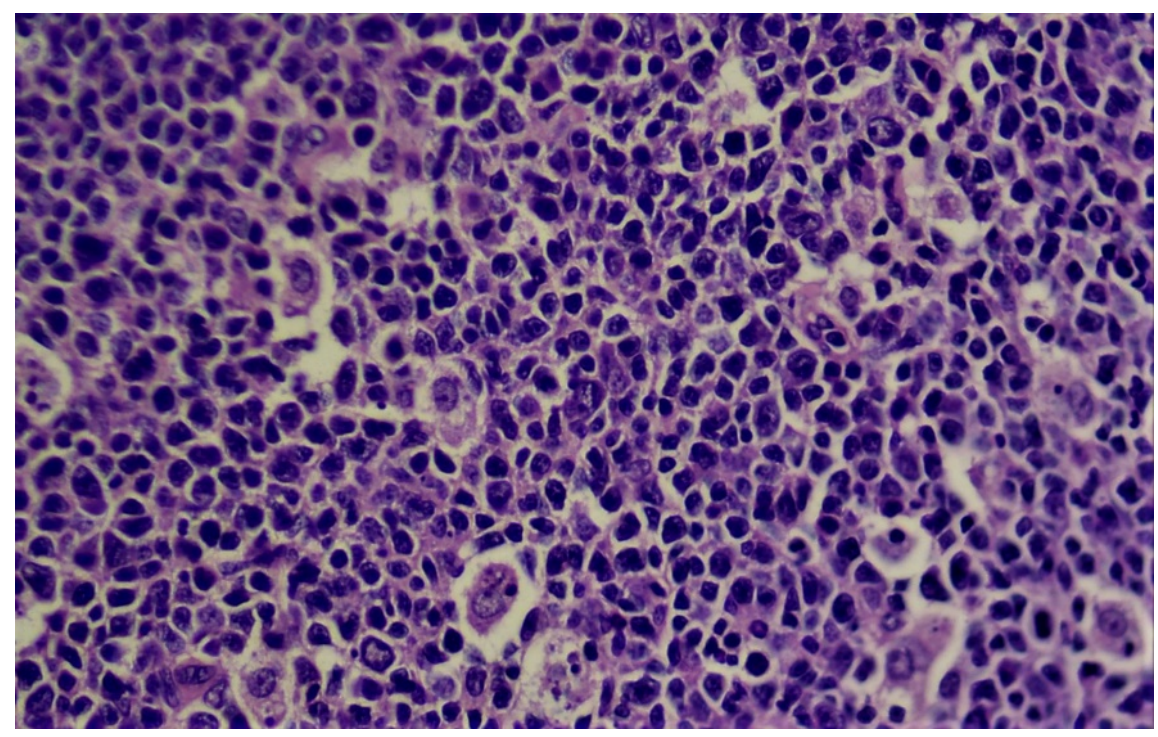

Figure 5: Slide section shows histiocytes exhibiting emperipolesis.

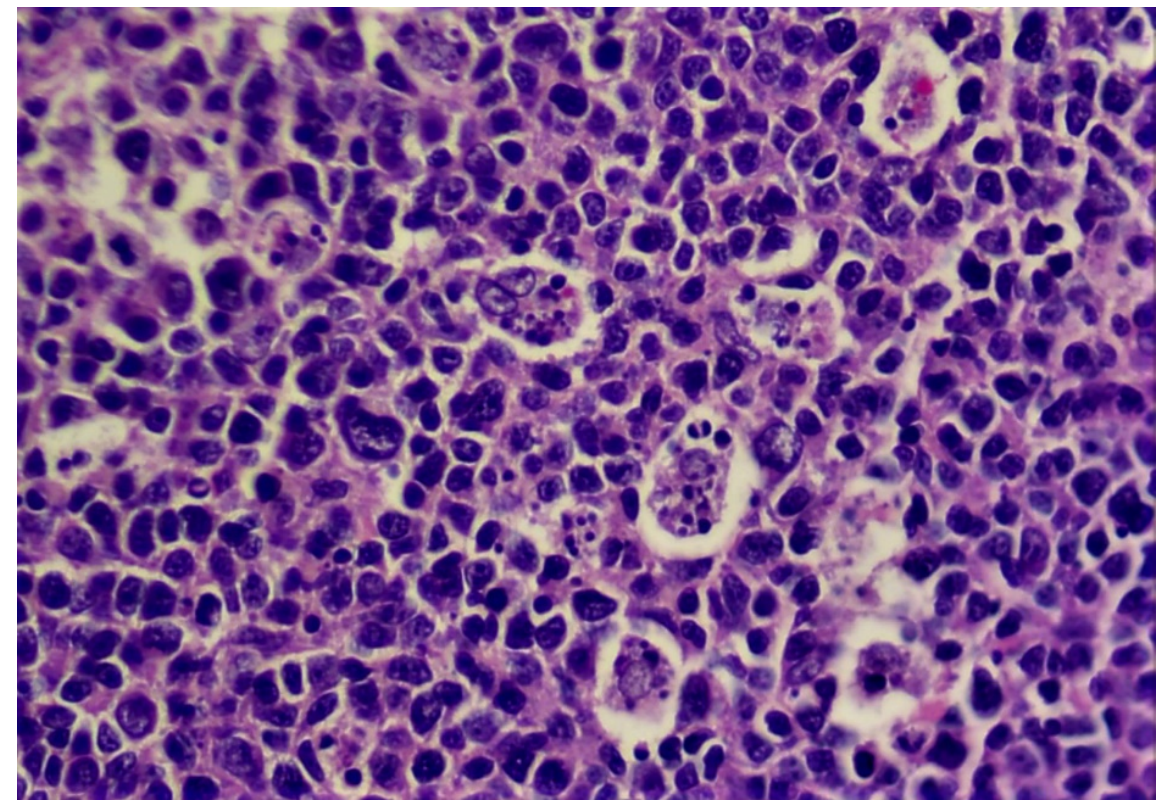

The patient was kept on steroids for 3 weeks with no residual disease and recurrence after 3-months of follow up. He was kept on Intramuscular injection. Betamethasone sodium (diprofos) $7 \mathrm{mg}$ stat and then tabs prednisolone $20 \mathrm{mg}$ once daily for 5 days then $10 \mathrm{mg}$ once daily for the next 5 days and then $5 \mathrm{mg}$ once daily for the next 5 days.

\section{Discussion}

Rosai-Dorfman Disease is a rare disease of lymph nodes often associated with extra nodal pattern and its characterized by benign histiocytic proliferation. ${ }^{1,4-6,10,13,17}$

RDD is commonly encountered in children and young adults and the trend is marked in those at their first to second decades of life and with slight male preponderance. ${ }^{5,9,12,13}$ 
The disease tend to present with various clinical features such as painless massive enlargement of cervical lymph nodes that may be accompanied by leukocytosis and elevated ESR, fever and hypergammaglobulinemia. . $^{4,5,8,10,16}$

Patients with RDD may present with concurrent involvement of extra nodal and nodal sites and such phenomenon may be challenging in terms of establishing its diagnosis and thus RDD may be missed during the first encounter. ${ }^{3,12}$

To date, the exact aetiopathogenesis of RDD has remained to be idiopathic though an aberrant exaggerated immune response to infectious agents especially viral aetiology may lead to proliferation of the histiocytes. ${ }^{8,12,13}$

Available studies have depicted the presence of Human Herpes Virus 6 genome through in situ hybridization as well as the relationship with Klebsiella, Epstein-Barr virus, Brucella, or Cytomegalovirus as the possible underlying aetiology. ${ }^{10,12,13}$

The pathognomonic histocytopathological feature of RDD is the presence of a phenomenon known as emperipolesis which is characterized by numerous large histiocytes with abundant, pale cytoplasm having variable number of intact lymphocytes within the cytoplasm. . $^{3,7,15,17-19}$

Histocytopathologically; In patients with RDD, the background is polymorphous comprising of lymphocytes, plasma cells and occasional neutrophils and all these features were present in the collected cytology and histopathology specimens. ${ }^{3,7,18,19}$

Although the histocytomorphological features are typical, diagnostic difficulties may sometimes arise. The major differential diagnosis of RDD upon FNAC of the lymph nodes or open lymph node biopsy include reactive lymphoid hyperplasia with sinus histiocytosis, Langerhans cell histiocytosis (LCH), Tuberculosis and Lymphoma which may be Hodgkin's or Non-Hodgkin's lymphoma. 3,7,17-19

Regarding the differentials of RDD; Reactive sinus histiocytosis tend to show loose clusters of histiocytes, accompanied by germinal center cells, reactive lymphocytes, immunoblasts, and tingible body macrophages without emperipolesis. Such cytologic findings may sometimes mislead the cytologist for a simple diagnosis of reactive lymphadenopathy. ${ }^{3,10,11,18,19}$

Cytologically; Langerhans cells have grooved nuclei and the background shows eosinophilic microabscess and Immunoreactivity with CD1a tends to be positive and thus can differentiate RDD. ${ }^{3,18,19}$ Tuberculous lymphadenitis shows epithelioid cell granuloma with or without caseous necrosis which are absent in RosaiDorfman disease. ${ }^{3,15,19}$ Hemophagocytic syndromes should be differentiated from Rosai-Dorfman disease on the basis of the presence of hemophagocytosis, absence of emperipolesis, and the presence of pancytopenia and hepatosplenomegaly. ${ }^{3,7}$

Hodgkin's disease and some variants of Non-Hodgkin's lymphoma show lymphocytes, plasma cells, histiocytes, eosinophils, and Reed- Sternberg cells. ${ }^{7,11,17}$

Histologically, apart from the mentioned known differential diagnoses, Gaucher's disease, metastatic carcinoma and melanoma and histiocytic sarcoma can add up to other differentials though the classical histomorphology of histiocytic proliferation inclusive of emperipolesis can lead to a definitive diagnosis. ${ }^{19}$ Whenever a pathologist is in doubt then, immunohistochemistry with CD11, CD14, CD34, CD68 and S-100 protein ${ }^{7,13,19}$ may be of help to support the diagnosis of RDD.

In our case, we established the diagnosis of RDD due to massive bilateral cervical and submandibular lymphadenopathy coupled with the presence of emperipolesis in histocytopathology.

Regarding the treatment of RDD, usually it has a self -limiting course in majority of the patients $4,5,8,10,12$ and therefore treatment is not necessary in majority of them. Our patient was treated using steroids which were provided by tapering the dose over two weeks for relief of symptoms and to reduce lymphadenopathy. Surgery is usually not required in patients with RDD ${ }^{4,5,8}$ unless the lymphadenopathy is too massive and 
causing discomfort to patient. It should generally be limited to biopsy for confirmation of the diagnosis similar to what was done to our patient where open lymph node biopsy was done for confirmation of the diagnosis..

Depending on the affected anatomical site, the treatment options include surgery, chemotherapy, steroids and radiotherapy. ${ }^{5,20}$ Medical therapy for RDD includes corticosteroids, cytotoxic agents or both. Combination of corticosteroids (prednisolone) along with agents like vinca alkaloids (vincristine and vinblastine), 6-mercaptopurine, methotrexate, or alkylating agent (cyclophosphamide) has shown promising outcomes. ${ }^{5,20}$ Generally, RDD has an indolent course with approximately $50 \%$ of them resolving without any residual disease. However in one-third of patients, residual adenopathy may be noted and remaining approximately $17 \%$ have unresolved symptoms even after $5-10$ years. ${ }^{5,21}$ In our case the patient showed no residual disease or recurrence at 3-months follow up.

\section{Conclusion}

While examining FNAC smears and biopsies of a lymph node, the possibility of RDD should always be kept in mind as one of the differential diagnoses. FNAC coupled by histopathology remains to be essential in establishing the diagnosis of RDD. However, FNAC is a reliable, minimally invasive, cost-effective, efficient and sensitive means to establish a conclusive diagnosis of RDD obviating the need for biopsy

\section{ACKNOWLEDGEMENT}

The patient is highly acknowledged for providing a written informed consent to publish the case report.

\section{CONFLICTS OF INTEREST}

None declared

\section{AUTHOR CONTRIBUTIONS}

ZSA: Performed open lymph node biopsy, collected information for the case and drafted the initial version of the manuscript. ZF and AIN both performed cytology and histopathology and also provided critical feedback of the manuscript. AAK: Drafted the initial version of the manuscript. FB: Performed open lymph node biopsy and provided critical feedback and editing of the manuscript. All authors read and approved the final version to be published in Clinical Case Reports

\section{ETHICS APPROVAL AND CONSENT TO PARTICIPATE}

This report is in accordance with the Declaration of Helsinki. The patient gave a written informed consent prior her inclusion in this report

\section{FUNDING}

None

\section{DATA AVAILABILITY}

The data that support the findings of this report are available from the corresponding author upon reasonable request.

\section{References}

1. Yadav S, Singhai A, Babu S. Rosai-Dorfman Disease of Extranodal Site. Annals of International Medical and Dental Research.;3(5):7.

2. Aziz M, Ray PS, Haider N, Rathore SP. Diagnosis of Rosai-Dorfman disease in elderly female on fine needle aspiration cytology: A case report. Case reports in pathology. 2012 Oct 14;2012.

3. Kataria SP, Sucheta GS, Sen R. Rosai Dorfman Disease Mimicking Lymphoma: A Case Report. 2016;6(October):293-5. 
4. Danisious T, Hettiarachchi M, Dharmadasa C, Jayaweera H. Rosai-Dorfman disease with renal involvement and associated autoimmune haemolytic anaemia in a 12-year-old girl: A case report. BMC pediatrics. 2020 Dec;20(1):1-5.

5. Ashish G, Chandrashekharan R, Parmar H. Rare case of Rosai Dorfman disease involving paranasal sinuses in paediatric patient: A case report. Egyptian Journal of Ear, Nose, Throat and Allied Sciences. 2016 Mar 1;17(1):43-6.

6. Stastny JF, Wilkerson ML, Hamati HF, Kornstein MJ. Cytologic features of sinus histiocytosis with massive lymphadenopathy. A report of three cases. Acta cytologica. 1997 May 1;41(3):871-6.

7. Deshpande AH, Nayak S, Munshi MM. Cytology of sinus histiocytosis with massive lymphadenopathy (Rosai-Dorfman disease). Diagnostic cytopathology. 2000 Mar 1;22(3):181-5.

8. Shi Y, Griffin AC, Zhang PJ, Palmer JN, Gupta P. Sinus histiocytosis with massive lymphadenopathy (Rosai-Dorfman Disease): A case report and review of 49 cases with fine needle aspiration cytology. Cytojournal. 2011;8.

9. Miceli A, Cleaver N, Spizuoco A. Rosai-Dorfman disease. Cutis. 2015 Jul 1;96(1):16-39.

10. Kushwaha R, Ahluwalia C, Sipayya V. Diagnosis of sinus histiocytosis with massive lymphadenopathy (Rosai-Dorfman Disease) by fine needle aspiration cytology. Journal of Cytology/Indian Academy of Cytologists. 2009 Apr;26(2):83.

11. Foucar E, Rosai J, Dorfman R. Sinus histiocytosis with massive lymphadenopathy (Rosai-Dorfman disease): review of the entity. InSeminars in diagnostic pathology 1990 Feb 1 (Vol. 7, No. 1, pp. 19-73).

12. Biswal P, Dhal I, Kar A, Nayak M, Pradhan D, Naik S. Diagnosis of Multifocal Extranodal Rosai Dorfman Disease by Fine Needle Aspiration Cytology. Journal of Advances in Medicine and Medical Research. 2015 Apr 3:872-6.

13. Amaraweera P, Perera HA. A case of Rosai Dorfman disease. Galle Medical Journal. 2009 Sep 28;11(1).

14. Santra G, Das BK, Mandal B, Kundu SS, Bandopadhyay A. Rosai-Dorfman disease. Singapore Med J. 2010 Oct 1;51(10):173-5.

15. Dar NH, Haque F, Hasan SA. Sinus Histiocytosis with Massive Lymphadenopathy. Indian Journal of Otolaryngology and Head and Neck Surgery. 2006 Jul 1;58(3):277-9.

16. Das DK, Gulati A, Bhatt NC, Sethi GR. Sinus histiocytosis with massive lymphadenopathy (RosaiDorfman disease): Report of two cases with fine-needle aspiration cytology. Diagnostic cytopathology. 2001 Jan;24(1):42-5.

17. Dhameja N, Chaudhary D, Bharti S, Awasthi C. Fnac of Rosai - Dorfman Disease - Report of 5 Cases. Ann Appl Bio-Sciences. 2018;5(2):C4-6.

18. Rastogi V, Sharma R, Misra SR, Yadav L, Sharma V. Emperipolesis-a review. Journal of clinical and diagnostic research: JCDR. 2014 Dec;8(12):ZM01.

19. Mallick S, Ghosh R, Iyer VK, Jain D, Mathur SR. Cytomorphological and morphometric analysis of 22 cases of Rosai-Dorfman disease: a large series from a tertiary care centre. Acta cytologica. 2013;57(6):625-32.

20. Jabali Y, Smrcka V, Pradna J. Rosai-Dorfman disease: successful long-term results by combination chemotherapy with prednisone, 6-mercaptopurine, methotrexate, and vinblastine: a case report. International journal of surgical pathology. 2005 Jul;13(3):285-9.

21. Raveenthiran V, Dhanalakshmi M, Rao PH, Viswanathan P. Rosai-Dorfman disease: report of a 3year-old girl with critical review of treatment options. European journal of pediatric surgery. 2003 Oct;13(05):350-4. 